\title{
Erratum: Vibrational Frequencies of Cerium-Oxide-Bound CO: A Challenge for Conventional DFT Methods [Phys. Rev. Lett. 125, 256101 (2020)]
}

Pablo G. Lustemberg, Philipp Plessow, Yuemin Wang, Chengwu Yang, Alexei Nefedov, Felix Studt, Christof Wöll, and M. Verónica Ganduglia-Pirovano®

(Received 29 November 2021; published 6 January 2022)

DOI: 10.1103/PhysRevLett.128.019901

In this Letter, Fig. 2(c) mistakenly shows the HSE06 isosurface of the charge density difference instead of that obtained with $\mathrm{PBE}+U$.

The authors state that the mistake does not change the scientific conclusions of the article in any way since the discussion in the text, as published, is correct.

PBE +U

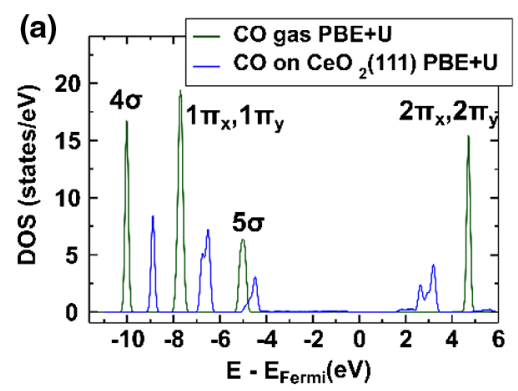

(c)

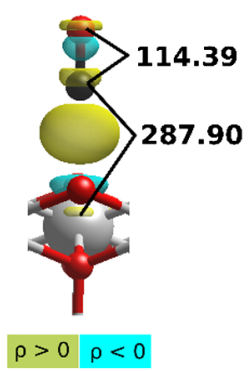

HSE06

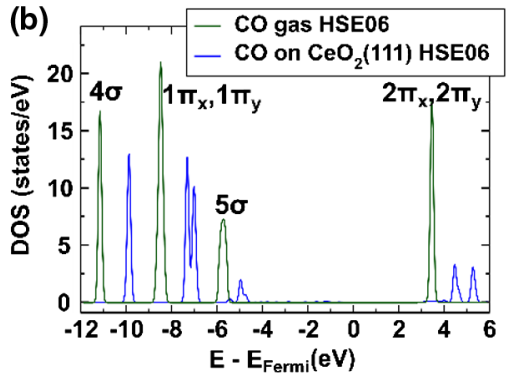

(d)

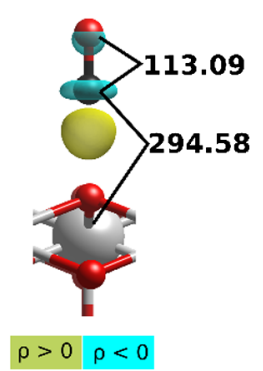

FIG. 2. Total density of states (DOS) projected onto the $\mathrm{CO}$ (blue) for $1 \mathrm{ML} \mathrm{CO}$ adsorbed on the $\mathrm{CeO}_{2}(111)$ surface in the atop Ce ${ }^{4+}$ configuration with the (a) PBE $+U(4.5)$ and (b) HSE06 approaches compared to that of CO in the gas phase. (c),(d) Isosurfaces of the charge density difference, namely, that of the $\mathrm{CO}$ adsorbed system from which both the charge density of the clean $\mathrm{CeO}_{2}(111)$ surface (with a structure corresponding to that of the adsorbed system) and that of the layer of adsorbed CO molecules have been subtracted. Selected interatomic distances (in pm) are indicated. 